\title{
Quantification of Task-Specific Glucose Metabolism with Constant Infusion of ${ }^{18}$ F-FDG
}

\author{
Andreas Hahn ${ }^{1}$, Gregor Gryglewski ${ }^{1}$, Lukas Nics ${ }^{2}$, Marius Hienert ${ }^{1}$, Lucas Rischka ${ }^{1}$, Chrysoula Vraka ${ }^{2}$, \\ Helen Sigurdardottir ${ }^{1}$, Thomas Vanicek ${ }^{1}$, Gregory M. James ${ }^{1}$, René Seiger ${ }^{1}$, Alexander Kautzky ${ }^{1}$, Leo Silberbauer ${ }^{1}$, \\ Wolfgang Wadsak ${ }^{2}$, Markus Mitterhauser ${ }^{2}$, Marcus Hacker ${ }^{2}$, Siegfried Kasper ${ }^{1}$, and Rupert Lanzenberger ${ }^{1}$ \\ ${ }^{I}$ Department of Psychiatry and Psychotherapy, Medical University of Vienna, Vienna, Austria; and ${ }^{2}$ Department of Biomedical \\ Imaging and Image-Guided Therapy, Division of Nuclear Medicine, Medical University of Vienna, Vienna, Austria
}

The investigation of cerebral metabolic rate of glucose (CMRGlu) at baseline and during specific tasks previously required separate scans with the drawback of high intrasubject variability. We aimed to validate a novel approach to assessing baseline glucose metabolism and task-specific changes in a single measurement with a constant infusion of ${ }^{18} \mathrm{~F}-\mathrm{FDG}$. Methods: Fifteen healthy subjects underwent two PET measurements with arterial blood sampling. As a reference, baseline CMRGlu was quantified from a $60-\mathrm{min}$ scan after ${ }^{18} \mathrm{~F}-\mathrm{FDG}$ bolus application using the Patlak plot (eyes closed). For the other scan, a constant radioligand infusion was applied for $95 \mathrm{~min}$, during which the subjects opened their eyes at 10-20 min and 60-70 min and tapped their right thumb to their fingers at 35-45 $\mathrm{min}$ and 85-95 min. The constant-infusion scan was quantified in two steps. First, the general linear model was used to fit regional time-activity curves with regressors for baseline metabolism, task-specific changes for the eyes-open and finger-tapping conditions, and movement parameters. Second, the Patlak plot was used for quantification of CMRGlu. Multiplication of the baseline regressor by $\beta$-values from the general linear model yielded regionally specific time-activity curves for baseline metabolism. Further, taskspecific changes in metabolism are directly proportional to changes in the slope of the time-activity curve and hence to changes in CMRGlu. Results: Baseline CMRGlu from the constant-infusion scan matched that from the bolus application (test-retest variability, $1.1 \% \pm 24.7 \%$ ), which was not the case for a previously suggested approach (variability, $-39.9 \% \pm 25.2 \%, P<0.001$ ). Task-specific CMRGlu increased in the primary visual and motor cortices for eyes open and finger tapping, respectively $(P<0.05$, familywise errorcorrected), with absolute changes of up to $2.1 \mu \mathrm{mol} / 100 \mathrm{~g} / \mathrm{min}$ and $6.3 \%$ relative to baseline. For eyes open, a decreased CMRGlu was observed in default-mode regions $(P<0.05$, familywise errorcorrected). CMRGlu quantified with venous blood samples $(n=6)$ showed excellent agreement with results obtained from arterial samples $(r>0.99)$. Conclusion: Baseline glucose metabolism and taskspecific changes can be quantified in a single measurement with constant infusion of ${ }^{18} \mathrm{~F}-\mathrm{FDG}$ and venous blood sampling. The high sensitivity and regional specificity of the approach offer novel possibilities for functional and multimodal brain imaging.

Received Mar. 23, 2016; revision accepted Jun. 7, 2016.

For correspondence or reprints contact: Rupert Lanzenberger, Department of Psychiatry and Psychotherapy, Medical University of Vienna, Waehringer Guertel 18-20, 1090 Vienna, Austria.

E-mail: rupert.lanzenberger@meduniwien.ac.at

Published online Jul. 7, 2016.

COPYRIGHT (c) 2016 by the Society of Nuclear Medicine and Molecular Imaging, Inc.
Key Words: glucose metabolism; quantification; task; positron emission tomography

J Nucl Med 2016; 57:1933-1940

DOI: 10.2967/jnumed.116.176156

$\mathbf{T}$ he investigation of glucose metabolism in the human brain represents an important tool for the assessment of brain function and alterations thereof. These include tumor imaging (1), neurologic diseases such as epilepsy (2) and Alzheimer disease (3-5), and psychiatric disorders such as major depression (6). Hence, PET using the radiolabeled glucose analog ${ }^{18} \mathrm{~F}$-FDG offers an excellent opportunity for imaging and quantification of glucose metabolism. ${ }^{18} \mathrm{~F}-\mathrm{FDG}$ is considered an irreversible radioligand since it is trapped in the early metabolic pathway and dephosphorylation is energetically less favorable $\left(k_{4} \neq 0\right.$, described in Materials and Methods section and Discussion section) $(7,8)$. After a bolus application, the uptake of the radioligand is rather stable after $30 \mathrm{~min}$, enabling simplified methods such as SUVs and ratios to be used in clinical routine for the assessment of baseline metabolism.

Similarly, if a certain task is performed in the early uptake period, task-specific glucose metabolism can be quantified (8). Several applications have been proven successful in this context. These include changes in the visual cortex after visual stimulation (9-11), changes in amygdala metabolism when a subject is watching videos that evoke emotion (12), and changes during more complex paradigms such as passive problem solving (13) and car driving (14). However, the differentiation of task-dependent changes from baseline metabolism in these studies required two separate PET scans, introducing the issue of considerable intrasubject variability between repeated scans, commonly reaching $25 \%$ if the scans are several days apart $(15,16)$. Therefore, the acquisition of baseline and task-specific metabolism in a single scan is highly desirable to increase both sensitivity and specificity.

One solution to the problem of repeated scans is use of a multipleinjection protocol to identify different physiologic states. In such a protocol, radioligand applications are temporally separated by 30$70 \mathrm{~min}$ in one continuous measurement (17-19). This, however, implies that imaging of each state and potential task performance consumes the same time, making it difficult to study task-related effects. Furthermore, the increase in scanning time and accompanied movement artifacts may limit the number of reasonably achievable 
states to two. In view of the sophisticated modeling requirements together with the increased radiation burden for subjects, the approach did not become a popular solution.

Recently, a novel technique with constant infusion of ${ }^{18} \mathrm{~F}$ FDG was proposed (20). In this technique, the radioligand is constantly applied during the entire scan, enabling assessment of dynamic task-specific changes in glucose metabolism. The same concept was proposed earlier, although in a different context, using simulations. It was shown that a certain task may change radioligand uptake during the experiment, but constant infusion was not considered at that time (21). The advantage of constant radioligand infusion is, however, its circumvention of the issue that ${ }^{18} \mathrm{~F}-\mathrm{FDG}$ uptake is most prominent early after a bolus injection and shows little change thereafter. Hence, a continuous supply of ${ }^{18}$ F-FDG provides free radioligand available to bind according to the metabolism at hand throughout the experiment.

Compared with well-established protocols using ${ }^{15} \mathrm{O}-\mathrm{H}_{2} \mathrm{O}$ or functional MRI, the assessment of neuronal activation with ${ }^{18}$ F-FDG may offer various benefits. The first two approaches quantify blood flow and the level of blood oxygenation, respectively, and are thus dependent on hemodynamics and neurovascular coupling. On the other hand, ${ }^{18} \mathrm{~F}$-FDG is a surrogate for glucose metabolism. The different imaging modalities are therefore measuring different aspects of the energy budget, namely oxygen supply versus glucose utilization, with the assets and drawbacks discussed elsewhere (8). This complementary nature of the different approaches may be particularly interesting in multimodal imaging studies using hybrid PET/MR systems (22). Furthermore, ${ }^{15} \mathrm{O}-\mathrm{H}_{2} \mathrm{O}$ studies are technically more challenging and functional MRI requires MR compatibility; hence, ${ }^{18} \mathrm{~F}-\mathrm{FDG}$ may represent a useful alternative.

Although the previous study provided the proof of principle, only 3 subjects were evaluated, and comparison with the commonly applied bolus application of ${ }^{18} \mathrm{~F}-\mathrm{FDG}$ was missing (20). Therefore, we aimed to validate the technique of constant radioligand infusion for the quantification of glucose metabolism at baseline and during two different tasks in a single PET measurement.

\section{MATERIALS AND METHODS}

\section{Subjects}

Fifteen right-handed healthy subjects participated in this study (mean age $\pm \mathrm{SD}, 24.5 \pm 4.7 \mathrm{y} ; 8$ women and $7 \mathrm{men}$ ). To rule out severe physical or mental disorders, all subjects were assessed by an experienced psychiatrist using the Structural Clinical Interview for DSM-IV. Additionally, they underwent routine medical examinations at the initial screening visit, which included blood laboratory testing, electrocardiography, and assessment of general physical and neurologic status (23). Female participants underwent a urine pregnancy test before each PET scan. The subjects reported no past or current substance abuse. All provided written informed consent after receiving a detailed explanation of the study protocol. The study was approved by the Ethics Committee of the Medical University of Vienna (ethics number 1916/2013), and the procedures were performed according to the Declaration of Helsinki.

\section{Study Design}

All subjects underwent two PET measurements in randomized order $(6.1 \pm 21.9 \mathrm{~d}$ apart). First, a 60 -min measurement was performed using a bolus application in which the subjects wore an eye mask to keep their eyes closed and were instructed to stay awake. This scan served as a reference to validate the constant-infusion approach. The second measurement lasted for $95 \mathrm{~min}$ and was done with a constant radioligand infusion for the entire measurement (Volumed $\mu$ VP7000; Arcomed). The infusion speed was set to $36 \mathrm{~mL} / \mathrm{h}(20)$. During this scan, the subjects opened their eyes at 10-20 min and 60-70 min after injection start and repeatedly tapped their right thumb to their fingers at 3545 min and 85-95 min after injection start, with the eyes closed and no finger movement at other times. None of the subjects reported falling asleep during the bolus or infusion scan.

\section{PET and Blood Sampling}

${ }^{18}$ F-FDG was freshly prepared every day according to a wellestablished method (24) using the E FASTlab platform (GE Healthcare). PET measurements were performed using an Advance (GE Healthcare) PET scanner as described previously (23). Briefly, head movement was minimized by placing each subject's head in a cushioned polyurethane bowl with straps around the forehead. An attenuation correction scan was performed for $5 \mathrm{~min}$ with retractable ${ }^{68} \mathrm{Ge}$ rod sources before radioligand injection. The dynamic PET scans were acquired in 3-dimensional mode with an intravenous (bolus or constant infusion) $3 \mathrm{MBq} / \mathrm{kg}$ injection of ${ }^{18} \mathrm{~F}-\mathrm{FDG}$. For the constant infusion, no initial bolus was applied and the infusion rate corresponded to $1.895 \mathrm{MBq} / \mathrm{kg} / \mathrm{h}$. PET images were reconstructed to consecutive frames of $1 \mathrm{~min}$. The spatial resolution of reconstructed images was $4.36 \mathrm{~mm}$ full width half maximum at $1 \mathrm{~cm}$ from the center of the field of view (voxel size, $3.125 \times 3.125 \times 4.25 \mathrm{~mm})$.

\section{Blood Sampling}

The glucose concentration in blood was measured before each PET scan. For the arterial input function, arterial blood samples were taken from the radial artery (25) during the PET scans. Automatic sampling was done for the first $3 \mathrm{~min}$ at a rate of $4 \mathrm{~mL} / \mathrm{min}$ (Allogg). Manual samples were acquired at 5, 10, 20, 40, and $60 \mathrm{~min}$ for the bolus scan or at $10,20,35,45,60,70,85$, and $95 \mathrm{~min}$ for the constant-infusion scan. For manual samples, whole-blood activity was measured in a $\gamma$-counter (Wizard ${ }^{2}, 3$ "; Perkin Elmer), and plasma was separated by centrifugation and again measured. The arterial input function was constructed by combining whole-blood activities from automated and manual sampling, which were further multiplied by average plasmato-whole-blood ratios. In addition, for 6 constant-infusion scans, manual venous blood samples were obtained from the cubital vein simultaneously with the arterial samples and were processed in the same way.

\section{MRI}

A T1-weighted structural MRI scan was acquired for all subjects (mMR; Siemens) and used for spatial normalization (magnetizationprepared rapid gradient-echo sequence; echo time/repetition time, 4.2/ 2,000 ms; voxel size, $1 \times 1 \times 1.1 \mathrm{~mm}$ ).

\section{Data Preprocessing and Regions of Interest}

Data were processed in SPM12 with default parameters unless specified otherwise. PET images were corrected for head motion (quality, 1), and the mean image was coregistered to the structural MR images (normalized mutual information). The structural MR images were then spatially normalized to MNI (Montreal Neurologic Institute) space via segmentation (26), and the obtained transformation matrices were applied to the dynamic PET images. Spatially normalized PET images were smoothed with an $8 \times 8 \times 8 \mathrm{~mm}$ gaussian kernel. Regions of interest (ROI) were taken from probabilistic atlases provided in FSL (http://fsl.fmrib.ox. ac.uk/) and included frontal, temporal, parietal, occipital, cingulate, and somatosensory cortices; thalamus; caudate; putamen; amygdalahippocampus complex; and cerebellar gray matter. Regional timeactivity curves were obtained as averages across each region (Fig. 1). 


\section{Quantification with Bolus Application}

The bolus measurement was quantified in PMOD, version 3.703 (http://pmod.com/), for all ROIs. Here, the influx constant,

$$
K_{i}=\frac{K_{1} k_{3}}{k_{2}+k_{3}}
$$

was estimated with the Gjedde-Patlak plot (27) as

$$
\frac{\mathrm{C}_{\mathrm{T}(\mathrm{T})}}{\mathrm{C}_{\mathrm{P}(\mathrm{T})}}=K_{i} \frac{\int_{0}^{T} \mathrm{C}_{\mathrm{P}(\mathrm{t})} \mathrm{dt}}{\mathrm{C}_{\mathrm{P}(\mathrm{T})}}+\text { intercept }
$$

with $C_{T}$ and $C_{P}$ being concentrations in tissue and plasma, respectively (Fig. 1). The plot of $\mathrm{C}_{\mathrm{T}(\mathrm{T})} / \mathrm{C}_{\mathrm{P}(\mathrm{T})}$ versus integral/ $\mathrm{C}_{\mathrm{P}(\mathrm{T})}$ becomes linear after time $t^{*}$, that is, when the free compartment has reached its steady state. Hence, $K_{i}$ can then be estimated as the slope. The arterial input function was fitted with a sum of 3 exponentials from the peak onward (25). The cerebral metabolic rate of glucose was further calculated as

$$
\text { CMRGlu }=K_{i} \frac{\text { Glup }}{\text { LC }} \times 100,
$$

with Glu $\mathrm{P}_{\mathrm{P}}$ and $\mathrm{LC}$ being the glucose concentration in plasma and the lumped constant, respectively. Here, the lumped constant was set to $0.89(28,29)$, and although some studies may have used different values, this factor affects only the absolute values in cerebral metabolic rate of glucose (CMRGlu) but not $K_{i}$ or task-specific changes in glucose metabolism. The actual lumped constant is thus of little relevance as long as it is consistently used within a study. For comparison, $K_{i}$ and CMRGlu were also estimated using an unconstrained 2-tissue-compartment model with $k_{4} \neq 0$ (3).

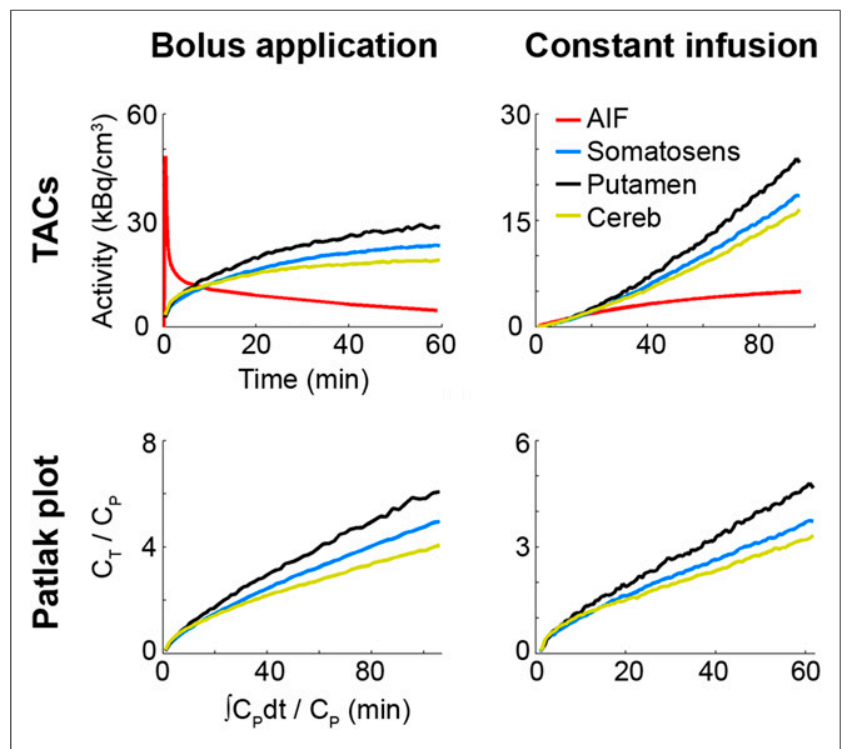

FIGURE 1. Time-activity curves (TACs) and corresponding Patlak plots for bolus application (baseline) and constant infusion (task) of ${ }^{18} \mathrm{~F}-\mathrm{FDG}$ from representative subject. Time-activity curves represent raw values and were extracted from somatosensory cortex, putamen, and cerebellum. For constant infusion, steady increase in activity was observed for all regions and to a lesser extent in blood. Transformation with Patlak plot demonstrated that linearity of slope is obtained quickly. $\mathrm{AlF}=$ arterial input function

\section{Quantification with Constant Infusion}

Before data analysis, PET images from constant-infusion measurements were masked with SPM12's gray matter prior converted into a binary code at 0.1 to exclude non-gray matter voxels from the analysis. Since ${ }^{18}$ F-FDG uptake occurs rather slowly with constant infusion and the paradigms lasted for $10 \mathrm{~min}$ each, sudden changes in time-activity curves were unlikely to be of physiologic origin. To reduce noise in PET signals (residual scatter, residual randoms, movement), a 12th-order finite impulse-response low-pass filter was applied with a cutoff frequency of $5 \mathrm{~min}$ (i.e., half the duration of the paradigm), which can be seen as a temporal smoothing.

Task-specific and baseline glucose metabolism from the constantinfusion measurement was quantified voxelwise in a 2-step procedure (Fig. 2) using Matlab R2011a (The MathWorks). The Discussion section presents the underlying assumptions. First, a general linear model (GLM) was used to fit each voxel's time-activity curve, which was adopted from functional MRI analyses (30).

$$
\begin{aligned}
Y= & \beta \times X+\varepsilon \\
\text { TAC }= & \beta_{\text {base }} \times \text { regressor }_{\text {base }}+\beta_{\text {eye }} \times \text { regressor }_{\text {eye }}+\beta_{\text {finger }} \\
& \times \text { regressor }_{\text {finger }}+\beta_{\text {move }} \times \text { regressor }_{\text {move }}+\varepsilon . \quad \text { Eq. } 4
\end{aligned}
$$

The GLM design matrix included 4 predictors, yielding voxelwise $\beta$-estimates for each regressor. One baseline regressor (yielding $\beta_{\text {base }}$ ) was defined individually as follows. Using linear regression, the average time course across all gray matter voxels was modeled by a third-order polynomial, whereas task effects were modeled as nuisance variables. Hence, the baseline regressor estimated by the polynomial was free of task effects. One regressor was included for each task $\left(\beta_{\text {eye }}\right.$ and $\left.\beta_{\text {finger }}\right)$, defined as a linear ramp function with slope of 1 during the task and slope of 0 otherwise (20). The task regressors were made orthogonal to the baseline regressor. Hence, the common variance is accounted for with the baseline regressor, whereas each task regressor models only the variance specific for each task. The fourth regressor was included to remove movement-related artifacts $\left(\beta_{\text {move }}\right)$. A principal component analysis was computed on the 6 motion parameters ( 3 translation and 3 rotation), and the first principal component score was used as a regressor (supplemental data; supplemental materials are available at http://jnm.snmjournals.org). In the second step, the Patlak plot was used to estimate $K_{i}$. Here, $\beta_{\text {base }}$ estimated from the GLM analysis was multiplied by the baseline regressor, which yielded regionally specific tissue time-activity curves for each voxel's baseline metabolism $\left(\mathrm{C}_{\mathrm{T} \text { base }}\right)$. As $K_{i}$ equals the slope in Equation 2 , baseline $K_{i \_ \text {base }}$ is thus directly proportional to $\mathrm{C}_{\mathrm{T}_{-} \text {base }}$

$$
K_{i_{-} \text {base }} \propto \mathrm{C}_{\mathrm{T}_{-} \text {base }} \propto \beta_{\text {base }} \times \text { regressor }_{\text {base }} .
$$

Further, task-specific changes in metabolism are proportional to changes in the slope of the time-activity curve (20), hence changing $\beta_{\text {base }}$ by the value of $\beta_{\text {task }}$ :

$$
K_{i_{-} \text {total }} \propto\left(\beta_{\text {base }}+\beta_{\text {task }}\right) \times \text { regressor }_{\text {base }},
$$

from which it follows that task-specific metabolism is proportional to

$$
K_{i_{-} \text {task }} \propto \beta_{\text {task }} \times \text { regressor }_{\text {base }} .
$$

The arterial input function for the Patlak plot was fitted with a sum of two exponentials as

$$
\mathrm{AIF}_{\text {fit }}=a_{1} \times\left(1-e^{-\frac{t}{\tau_{1}}}\right)+a_{2} \times\left(1-e^{-\frac{t}{\tau_{2}}}\right),
$$

with $a$ and $\tau$ representing estimated parameters (Figs. 1 and 2). t* was fixed to $30 \mathrm{~min}$ for simplicity. 


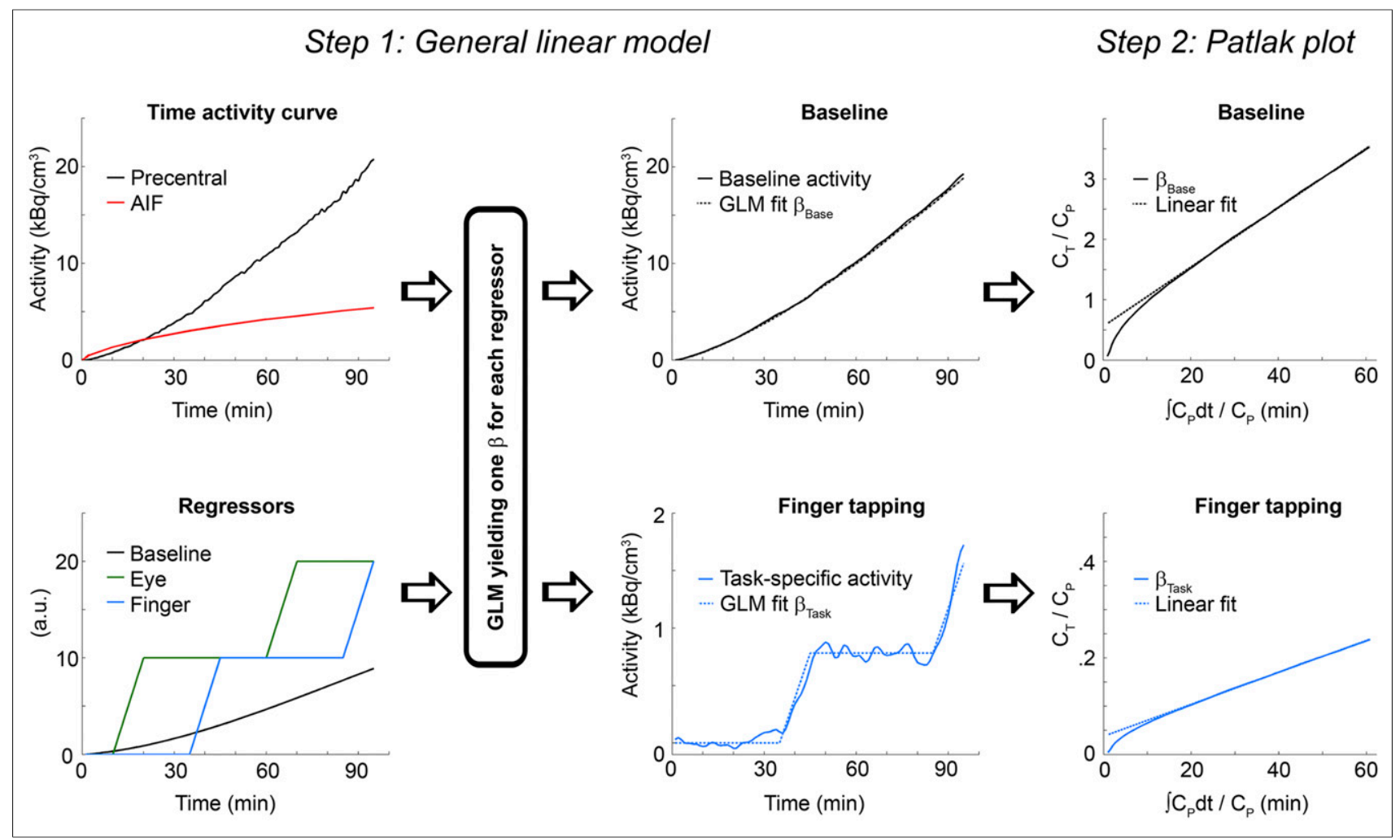

FIGURE 2. Schematic workflow of quantification with constant infusion. Group-averaged data from representative brain region with significantly increased glucose metabolism are shown, namely left precentral cortex during right-finger tapping $(P<0.05$, FWE-corrected voxel level; Fig. 4). First, time-activity curve is fitted with GLM using regressors for baseline metabolism as well as eyes-open condition and finger-tapping task. Estimated $\beta$-values from GLM demonstrate adequate fits of baseline and task-specific activity (dashed lines). Second, Patlak plot is applied to quantify glucose metabolism. Here, multiplication of baseline regressor with $\beta$-values yields time-activity curves $\left(\mathrm{C}_{\mathrm{T}}\right)$ and combination with arterial input function (AIF, $\mathrm{C}_{\mathrm{P}}$ ) enables estimation of influx constant $K_{i}$ as slope.

To evaluate the potential substitution of arterial blood samples by venous ones, baseline and task-specific $K_{i}$ also was computed with venous blood samples using the same procedure.

For comparison, we also estimated baseline $K_{i}$ and CMRGlu from the previously suggested approach (20). Basically, this included only the first part of the above-described method, namely the linear
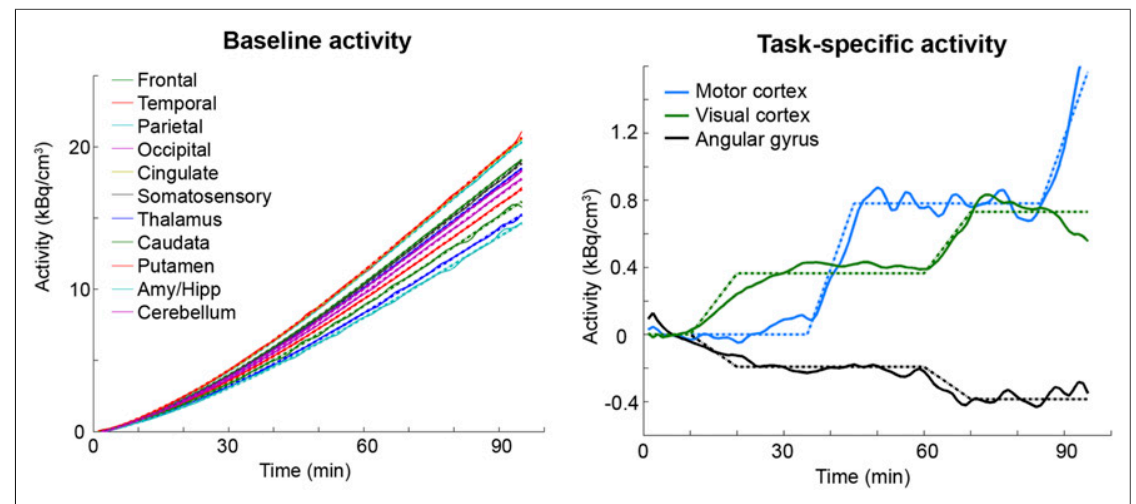

FIGURE 3. Baseline and task-specific metabolism obtained from constant-infusion approach (solid lines). Corresponding results obtained from GLM (dashed lines) show model fits across different brain regions for baseline and task metabolism. Baseline activity was obtained by subtracting all regressors $\beta$ from raw time-activity curve except that of baseline; task-specific activity was calculated by subtracting all regressors ${ }^{\star} \beta$ from raw time-activity curve except that of actual task (Eq. 4). Task-specific changes are exemplarily shown for eyes-open condition (with increases in primary visual cortex and decreases in angular gyrus) and for finger-tapping task (with increases in primary motor cortex). regression but without the Patlak plot analysis. Hence, $K_{i}$ was previously defined as the slope of $\mathrm{C}_{\mathrm{T}(\mathrm{T})} / \mathrm{C}_{\mathrm{P}(\mathrm{T})}$ versus time.

\section{Statistics}

The bias of baseline $K_{i}$ between the Patlak plot and the 2-tissuecompartment model was calculated by regression analysis, that is, evaluating slope and intercept across all ROIs. For validation of the constant-infusion approach, the estimated baseline $K_{i}$ and CMRGlu were compared with those from the bolus application. Test-retest values were calculated for each ROI as

$$
\begin{aligned}
& \left(K_{i \_ \text {bolus }}-K_{i \_ \text {infusion }}\right) / \\
& \quad\left[\left(K_{i \_ \text {bolus }}+K_{i \_ \text {infusion }}\right) / 2\right] \times 100 .
\end{aligned}
$$

Test-retest values were then compared with the original approach (20) by repeatedmeasures ANOVA with region and method as fixed factors and subject as random factor. Significant changes in task-related glucose metabolism were computed voxelwise in SPM12 using a 1-sample $t$ test for each of the tasks $(P<0.05$, familywise error [FWE]-corrected). To evaluate the substitution of arterial blood samples by venous ones, $K_{i}$ and CMRGlu estimated with both blood samples were compared using linear 
regression implemented in Matlab across all ROIs plus 3 regions with task-relevant changes in metabolism.

\section{RESULTS}

For the bolus application, baseline $K_{i}$ values were generally lower when estimated with the Patlak plot than with the 2-tissuecompartment model (slope, 0.73; intercept, 0.0004). For the constant-infusion approach, baseline metabolism was adequately fitted with the GLM (Fig. 3) and was consistent with values from the bolus application (test-retest $K_{i}, 2.4 \% \pm 16.0 \%$; CMRGlu, $1.1 \% \pm 24.7 \%$; supplemental data). In contrast, the previous method (20) underestimated baseline metabolism (test-retest $K_{i},-39.0 \% \pm$ 17.0\%; CMRGlu, $-39.9 \% \pm 25.2 \%$ ), which, across all ROIs, was significantly different from the approach presented here $\left(F_{1,333}=608.4\right.$ and 241.4, both $P<0.001$, for $K_{i}$ and CMRGlu, respectively).

Task-specific increases of up to $2.1 \mu \mathrm{mol} / 100 \mathrm{~g} / \mathrm{min}$ in glucose metabolism were observed in the primary visual and primary motor cortices for the eyes-open condition and right-finger tapping, respectively, as well as in the cerebellum for both tasks (all $P<0.05$, FWE-corrected voxel level) (Figs. 3 and 4). In these regions, percentage signal changes reached $6.1 \% \pm$ $1.7 \%$ and $6.3 \% \pm 2.8 \%$, for the eyes-open condition and

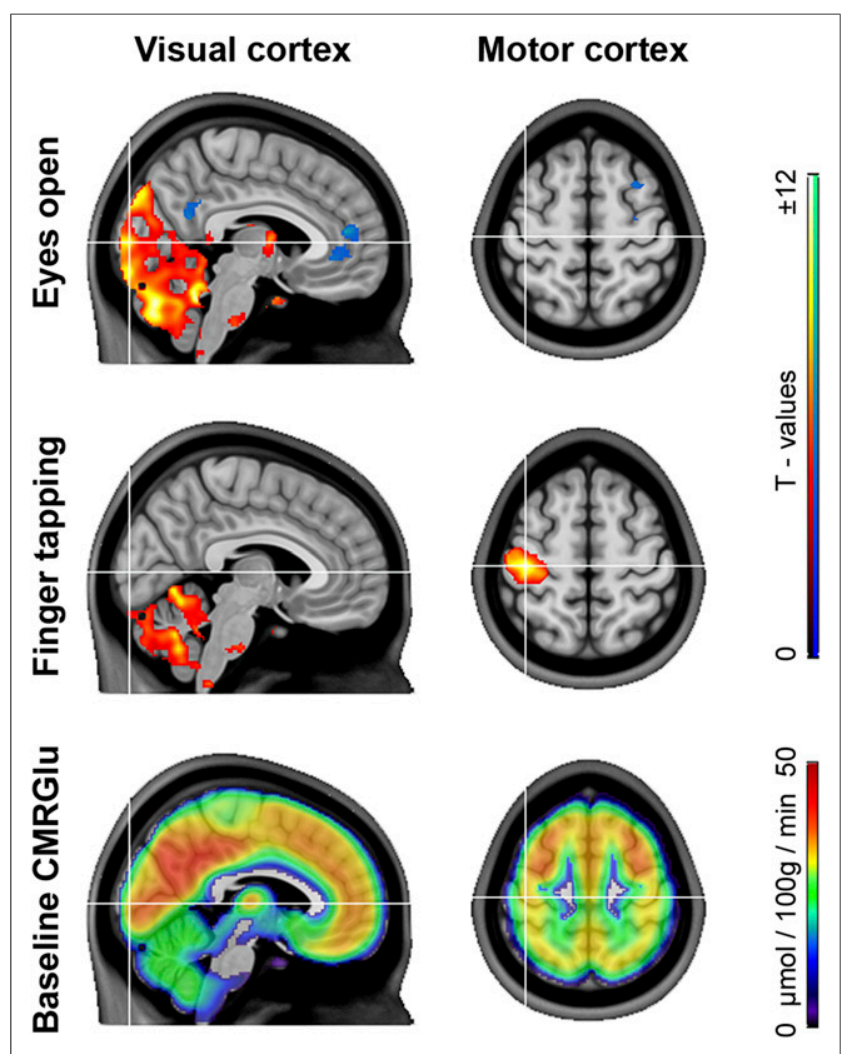

FIGURE 4. Task-specific changes in glucose metabolism during eyesopen condition and right-finger tapping (both $P<0.05$, cluster level). Increased CMRGlu was observed in primary visual cortex and primary motor cortex, respectively. Both tasks also showed increased CMRGlu in cerebellum. Decreased CMRGlu was observed in regions of defaultmode network when eyes were opened. Baseline CMRGlu is shown for comparison. right-finger tapping, respectively (Table 1). Conversely, no relevant changes were identified in the motor cortex during the eyes-open condition or in the visual cortex during finger tapping, even without correction for multiple comparisons ( $-0.4 \%$, Table 1). Interestingly, we also observed task-specific decreases in metabolism for the eyes-open condition, mostly in the frontal regions and the default-mode network (i.e., posterior cingulate and medial prefrontal cortices, Fig. 4; all $P<0.05$, FWE-corrected voxel or cluster level).

Although differences between arterial and venous samples were observed $(31,32)$, changes in $\int \mathrm{C}_{\mathrm{P}(\mathrm{t})} \mathrm{dt}$ (i.e., the factor determining $K_{i}$ in the steady state) were highly correlated between both blood pools $(r=0.99$; slope, 0.92 ; intercept, -0.19$)$. Hence, quantification with venous blood samples showed excellent agreement with the results from arterial samples. This was true for baseline $K_{i}$ and CMRGlu across all ROIs, as well as for increases in the primary visual cortex when the eyes were open, increases in the primary motor cortex during finger tapping, and decreases in the angular gyrus when the eyes were open (all $r>0.99$; slope, 0.99...1.04; intercept, 0..1.31).

\section{DISCUSSION}

In this work, we have shown that baseline and task-specific changes in glucose metabolism can be robustly estimated in a single measurement with constant infusion of ${ }^{18} \mathrm{~F}-\mathrm{FDG}$. Task-related changes were regionally and temporally specific for the eyes-open condition and right-finger tapping, enabling separation of different metabolic demands.

Compared with the previously introduced approach (20), several extensions and validations were included here. The combination of the GLM with the subsequent Patlak plot analysis markedly decreased test-retest variability for baseline metabolism. The exact difference from the previous approach is, however, difficult to estimate because the previous study did not explicitly specify the baseline term. Our comparison with the bolus-application scans validates the use of a constant infusion to robustly estimate baseline metabolism. Proceeding from this validation, task-specific changes could be quantified because these are proportional to changes in the slope of the time-activity curve. Interestingly, the estimated percentage change in signal $(\sim 6 \%)$ was lower than previously reported, possibly because of the simplicity of the tasks used here. For instance, light stimulation has been shown to produce $6 \%-12 \%$ increased metabolism in the visual cortex (9) and more than $20 \%$ during checkerboard stimulation $(9,20)$. Again, the fact that our stimuli were rather subtle underlines the sensitivity of the approach, whereas a more pronounced effect can be expected from paradigms with stronger stimuli. On the other hand, the changes in glucose metabolism were higher than those usually observed $(\sim 1 \%-2 \%(30))$ in functional MRI experiments using the blood-oxygen level-dependent effect, even for $7 \mathrm{~T}$ imaging (33). Given the complementary nature of different imaging techniques $(8,11,22,34,35)$, the presented approach may offer novel applications for simultaneous multimodal imaging with hybrid imaging systems. Finally, the excellent agreement that was found when venous and arterial blood samples were compared enables further simplification and thus greater accessibility of the method.

The approach also showed high regional specificity, as no metabolic changes in the visual cortex were observed during finger-tapping or in the motor cortex when eyes were opened. The increased glucose metabolism in the visual cortex is in line 
TABLE 1

Task-Specific Changes in Glucose Metabolism for Eyes-Open Condition and Right-Finger Tapping as Compared with Baseline

\begin{tabular}{|c|c|c|c|c|c|c|c|}
\hline Region & $x(\mathrm{~mm})$ & $y(\mathrm{~mm})$ & $z(\mathrm{~mm})$ & $t$ value & $\begin{array}{l}\text { CMRGlu baseline } \\
(\mu \mathrm{mol} / 100 \mathrm{~g} / \mathrm{min})\end{array}$ & $\begin{array}{c}\text { CMRGlu task } \\
(\mu \mathrm{mol} / 100 \mathrm{~g} / \mathrm{min})\end{array}$ & $\begin{array}{c}\text { Signal } \\
\text { change (\%) }\end{array}$ \\
\hline \multicolumn{8}{|l|}{ Eyes open $>$ baseline } \\
\hline Lingual L & -4 & -88 & -14 & $10.4^{*}$ & $30.5 \pm 5.4$ & $1.1 \pm 0.5$ & $3.6 \pm 1.4$ \\
\hline Intracalcarine L & -8 & -84 & 8 & $10.0^{*}$ & $30.6 \pm 5.2$ & $0.9 \pm 0.4$ & $3.0 \pm 1.1$ \\
\hline Supracalcarine R & 16 & -66 & 14 & $10.4^{*}$ & $33.8 \pm 5.8$ & $0.7 \pm 0.3$ & $2.2 \pm 0.8$ \\
\hline Occipital pole R & 8 & -96 & -8 & $9.8^{\star}$ & $28.2 \pm 6.3$ & $1.1 \pm 0.5$ & $4.0 \pm 1.9$ \\
\hline Cuneus & 0 & -82 & 26 & $11.8^{*}$ & $32.9 \pm 5.3$ & $0.8 \pm 0.3$ & $2.3 \pm 0.8$ \\
\hline Cerebellum crus II L & -12 & -88 & -24 & $17.2^{*}$ & $24.8 \pm 4.1$ & $1.5 \pm 0.5$ & $6.1 \pm 1.7$ \\
\hline Cerebellum crus II R & 8 & -80 & -28 & $9.4^{*}$ & $26.1 \pm 4.4$ & $1.5 \pm 0.7$ & $5.5 \pm 2.2$ \\
\hline Cerebellum VI L & -28 & -54 & -28 & $16.5^{\star}$ & $26.8 \pm 3.9$ & $0.8 \pm 0.2$ & $3.1 \pm 0.8$ \\
\hline Cerebellum crus I L & -26 & -88 & -30 & $11.9^{\star}$ & $18.7 \pm 3.5$ & $0.8 \pm 0.3$ & $4.0 \pm 1.5$ \\
\hline Cerebellum crus I R & 52 & -52 & -34 & $12.9^{*}$ & $18.3 \pm 3.5$ & $1.1 \pm 0.4$ & $5.8 \pm 1.8$ \\
\hline Cerebellum vermis IX L & -2 & -54 & -30 & $11.8^{*}$ & $20.4 \pm 2.8$ & $0.9 \pm 0.3$ & $4.2 \pm 1.5$ \\
\hline Hippocampus L & -30 & -18 & -12 & $9.7^{*}$ & $19.4 \pm 2.9$ & $1.0 \pm 0.4$ & $5.2 \pm 2.2$ \\
\hline \multicolumn{8}{|l|}{ Eyes open $<$ baseline } \\
\hline Frontal superior $\mathrm{L}$ & -18 & 32 & 46 & $-9.2^{*}$ & $33.2 \pm 6.7$ & $-0.7 \pm 0.4$ & $-1.9 \pm 0.8$ \\
\hline Central L & -40 & -24 & 58 & -0.7 & $32.9 \pm 5.3$ & $-0.2 \pm 0.8$ & $-0.4 \pm 2.2$ \\
\hline \multicolumn{8}{|l|}{ Finger tapping $>$ baseline } \\
\hline Central L & -40 & -24 & 58 & $11.5^{\star}$ & $32.9 \pm 5.3$ & $2.1 \pm 1.0$ & $6.3 \pm 2.8$ \\
\hline Precentral L & -32 & -16 & 70 & $10.7^{*}$ & $22.7 \pm 4.4$ & $1.3 \pm 0.6$ & $5.7 \pm 2.5$ \\
\hline Hippocampus L & -28 & -16 & -12 & $12.1^{*}$ & $19.4 \pm 3.1$ & $1.1 \pm 0.4$ & $5.6 \pm 1.9$ \\
\hline Cerebellum VI L & -32 & -56 & -26 & $13.0^{\star}$ & $29.6 \pm 4.3$ & $1.3 \pm 0.4$ & $4.3 \pm 1.3$ \\
\hline Cerebellum VI R & 8 & -64 & -10 & $11.3^{\star}$ & $26.0 \pm 3.6$ & $0.9 \pm 0.4$ & $3.5 \pm 1.3$ \\
\hline Cerebellum crus I R & 52 & -52 & -32 & $9.4^{*}$ & $19.7 \pm 4.0$ & $1.0 \pm 0.5$ & $5.0 \pm 2.2$ \\
\hline \multicolumn{8}{|l|}{ Finger tapping $<$ baseline } \\
\hline Intracalcarine L & -8 & -84 & 8 & -0.8 & $30.6 \pm 5.2$ & $-0.2 \pm 0.7$ & $-0.3 \pm 2.3$ \\
\hline
\end{tabular}

${ }^{*} P<0.05$, FWE-corrected voxel level.

Significance thresholds were $t=8.7$ for $P<0.05$, FWE-corrected, and $t=3.8$ for $P<0.001$, uncorrected. Coordinates and $t$ values were obtained from SPM analysis. Corresponding CMRGlu as obtained from Patlak plot is shown for baseline condition and each task as well as percentage signal changes from baseline.

with various previous studies $(9,11,35)$. In addition, increased metabolism in the cerebellum during the eyes-open condition and finger tapping may relate to the cerebellum's involvement in attention (36), visual processing (37), and motor control (38). Furthermore, we observed decreases in glucose metabolism in regions of the frontal cortex and of the default-mode network. Such a deactivation during task performance was first described with PET blood flow and oxygen extraction studies $(34,39)$ and in a marked number of functional MRI experiments thereafter (40). Interestingly, a recent study reported increased glucose metabolism when the eyes-open and eyes-closed conditions were compared (11). This disagreement with our finding is probably related to a difference in study design, namely recruitment of two different groups to evaluate two different states (11). Further considering an intrasubject variability of around $25 \%$ for repeated scans, we argue that the assessment of baseline and task-specific metabolism in a single measurement is essential for functional PET studies investigating the dynamic activation of distinct brain regions.

The method proposed here incorporates several assumptions and limitations. First, application of the Patlak plot does not take into account the fact that $k_{4}$ may actually be higher than 0 , resulting in a known underestimation of CMRGlu (here, 27\%) (3,29). Although this is important to keep in mind when different studies are compared, the underestimation is a scaling factor and may thus be less problematic if applied consistently across subjects. Further, the arterial input function did not reach equilibrium after bolus application or during constant infusion. For the Patlak plot, this is not required, as changes in arterial input function need to be only small enough to allow the reversible part to reach a steady state $(27,41)$. Here, the changes in arterial input function were $54 \% \pm 40 \%$ lower, and linearity was reached more quickly for constant infusion than for bolus, supporting the applicability of the Patlak plot. Another assumption 
is that baseline metabolism can be modeled by a single baseline regressor, which is multiplied by regional $\beta$-estimates. Although the baseline regressor was cleaned of task effects and reasonable fits were obtained for all investigated ROIs, the assumption needs further validation in populations with regional alterations such as brain tumors. Next, we assume that the relationship between the neuronal response and glucose metabolism is linear time-invariant, similar to functional MRI (30). In this context, linearity implies that changes in the neuronal response will convert to changes in metabolism by the same factor-a reasonable assumption given their tight coupling (8). Conversely, changes in metabolism by two simultaneous events will sum together and add to the baseline metabolism. Time invariance refers to the temporal coupling between the stimulus and the ${ }^{18} \mathrm{~F}-\mathrm{FDG}$ uptake. Although these assumptions are derived from functional MRI, PET imaging is less dependent on the prerequisite of neurovascular coupling, and ${ }^{18} \mathrm{~F}-\mathrm{FDG}$ uptake was previously unrelated to pronounced changes in blood flow (20). Hence, linear time invariance seems to be a valid simplification for the proposed method. Finally, the approach assumes that both baseline and task-specific metabolism are at a steady state and that taskrelated glucose consumption is constant throughout the task. As discussed previously (20), this steady-state assumption is violated by almost all functional imaging methods but is a necessary approximation to enable quantitative description. On the other hand, if a task does not elicit a constant change in metabolism, regressors with shapes different from a linear ramp function may yield a better estimate of task-specific glucose consumption.

As this study is among the first of its kind, the approach could potentially benefit from further advances. For instance, the combination of a bolus application plus a constant infusion may yield a better equilibrium of plasma activity and increase the signal-tonoise ratio. Another improvement would be not only to start but also to end the paradigm with a baseline condition to increase confidence in the task-specific model fits. Finally, a more explicit stimulation (in this case, for example, explicit pacing of finger tapping (42)) may yield more robust changes in CMRGlu. It remains to be established how well the approach performs during more complex paradigms, especially when different conditions are being compared (e.g., activations and deactivations during recognition of emotions or objects (33)). The detection of task-relevant decreases in CMRGlu gives confidence that the approach is sensitive enough for such contrasts.

\section{CONCLUSION}

We have demonstrated that glucose metabolism at baseline and during several tasks can be quantified simultaneously using a constant ${ }^{18}$ F-FDG infusion and venous blood sampling. Estimating these different aspects of glucose metabolism in a single measurement decreases the intrasubject variability inherent in repeated scans. In view of its high sensitivity to subtle stimuli together with its regional specificity, the method offers novel applications in functional brain imaging.

\section{DISCLOSURE}

The costs of publication of this article were defrayed in part by the payment of page charges. Therefore, and solely to indicate this fact, this article is hereby marked "advertisement" in accordance with 18 USC section 1734. Gregor Gryglewski is the recipient of a DOC Fellowship from the Austrian Academy of Sciences at the Institute of Psychiatry and Psychotherapy,
Medical University of Vienna. This research was supported by a grant from the Austrian Science Fund (FWF KLI 516) to Rupert Lanzenberger. This scientific project was performed with the support of the Medical Imaging Cluster of the Medical University of Vienna. No other potential conflict of interest relevant to this article was reported.

\section{ACKNOWLEDGMENTS}

We are grateful to the technical team of the PET Centre, especially Harald Ibeschitz, Ingrid Leitinger, and Volker Weiss. We thank the physicians-Anna Höflich, Arkadiusz Komorowski, Marie Spies, Pia Baldinger, Christoph Kraus, and Dietmar Winklerfor medical support.

\section{REFERENCES}

1. Meric K, Killeen RP, Abi-Ghanem AS, et al. The use of ${ }^{18}$ F-FDG PET ratios in the differential diagnosis of common malignant brain tumors. Clin Imaging. 2015;39:970-974.

2. Kerr WT, Nguyen ST, Cho AY, et al. Computer-aided diagnosis and localization of lateralized temporal lobe epilepsy using interictal FDG-PET. Front Neurol. 2013;4:31.

3. Devanand DP, Mikhno A, Pelton GH, et al. Pittsburgh compound B ( $\left.{ }^{11} \mathrm{C}-\mathrm{PIB}\right)$ and fluorodeoxyglucose $\left({ }^{18} \mathrm{~F}-\mathrm{FDG}\right) \mathrm{PET}$ in patients with Alzheimer disease, mild cognitive impairment, and healthy controls. J Geriatr Psychiatry Neurol. 2010;23:185198.

4. Mosconi L, Tsui WH, Rusinek H, et al. Quantitation, regional vulnerability, and kinetic modeling of brain glucose metabolism in mild Alzheimer's disease. Eur J Nucl Med Mol Imaging. 2007;34:1467-1479.

5. Ossenkoppele R, Tolboom N, Foster-Dingley JC, et al. Longitudinal imaging of Alzheimer pathology using $\left[{ }^{11} \mathrm{C}\right] \mathrm{PIB},\left[{ }^{18} \mathrm{~F}\right] \mathrm{FDDNP}$ and $\left[{ }^{18} \mathrm{~F}\right] \mathrm{FDG}$ PET. Eur $J$ Nucl Med Mol Imaging. 2012;39:990-1000.

6. McGrath CL, Kelley ME, Holtzheimer PE, et al. Toward a neuroimaging treatment selection biomarker for major depressive disorder. JAMA Psychiatry. 2013;70:821-829.

7. Wadsak W, Mitterhauser M. Basics and principles of radiopharmaceuticals for PET/CT. Eur J Radiol. 2010;73:461-469.

8. Kessler RM. Imaging methods for evaluating brain function in man. Neurobiol Aging. 2003;24(suppl 1):S21-S35.

9. Phelps ME, Kuhl DE, Mazziota JC. Metabolic mapping of the brain's response to visual stimulation: studies in humans. Science. 1981;211:1445-1448.

10. Vlassenko AG, Rundle MM, Mintun MA. Human brain glucose metabolism may evolve during activation: findings from a modified FDG PET paradigm. Neuroimage. 2006;33:1036-1041.

11. Riedl V, Bienkowska K, Strobel C, et al. Local activity determines functional connectivity in the resting human brain: a simultaneous FDG-PET/fMRI study. J Neurosci. 2014;34:6260-6266.

12. Cahill L, Haier RJ, Fallon J, et al. Amygdala activity at encoding correlated with long-term, free recall of emotional information. Proc Natl Acad Sci USA. 1996; 93:8016-8021.

13. Haier RJ, White NS, Alkire MT. Individual differences in general intelligence correlate with brain function during nonreasoning tasks. Intelligence. 2003; 31:429-441.

14. Jeong M, Tashiro M, Singh LN, et al. Functional brain mapping of actual cardriving using $\left[{ }^{18}\right.$ F]FDG-PET. Ann Nucl Med. 2006;20:623-628.

15. Schmidt ME, Ernst M, Matochik JA, et al. Cerebral glucose metabolism during pharmacologic studies: test-retest under placebo conditions. J Nucl Med. 1996; 37:1142-1149.

16. Brooks RA, Di Chiro G, Zukerberg BW, Bairamian D, Larson SM. Test-retest studies of cerebral glucose metabolism using fluorine-18 deoxyglucose: validation of method. J Nucl Med. 1987;28:53-59.

17. Chang JY, Duara R, Barker W, et al. Two behavioral states studied in a single PET/FDG procedure: error analysis. J Nucl Med. 1989;30:93-105.

18. Murase K, Kuwabara H, Yasuhara Y, Evans AC, Gjedde A. Mapping of change in cerebral glucose utilization using fluorine-18 fluorodeoxyglucose double injection and the constrained weighted-integration method. IEEE Trans Med Imaging. 1996;15:824-835. 
19. Nishizawa S, Kuwabara H, Ueno M, Shimono T, Toyoda H, Konishi J. Doubleinjection FDG method to measure cerebral glucose metabolism twice in a single procedure. Ann Nucl Med. 2001;15:203-207.

20. Villien M, Wey HY, Mandeville JB, et al. Dynamic functional imaging of brain glucose utilization using fPET-FDG. Neuroimage. 2014;100:192-199.

21. Huang SC, Phelps ME, Hoffman EJ, Kuhl DE. Error sensitivity of fluorodeoxyglucose method for measurement of cerebral metabolic rate of glucose. J Cereb Blood Flow Metab. 1981;1:391-401.

22. Wehrl HF, Hossain M, Lankes K, et al. Simultaneous PET-MRI reveals brain function in activated and resting state on metabolic, hemodynamic and multiple temporal scales. Nat Med. 2013;19:1184-1189.

23. Hahn A, Haeusler D, Kraus C, et al. Attenuated serotonin transporter association between dorsal raphe and ventral striatum in major depression. Hum Brain Mapp. 2014;35:3857-3866.

24. Hamacher K, Coenen HH, Stocklin G. Efficient stereospecific synthesis of no-carrier-added 2-[ $\left.{ }^{18} \mathrm{~F}\right]$-fluoro-2-deoxy-D-glucose using aminopolyether supported nucleophilic substitution. J Nucl Med. 1986;27:235-238.

25. Hahn A, Nics L, Baldinger $P$, et al. Combining image-derived and venous input functions enables quantification of serotonin-1A receptors with [carbonyl $-{ }^{11} \mathrm{C}$ ]WAY100635 independent of arterial blood sampling. Neuroimage. 2012;62:199-206.

26. Ashburner J, Friston KJ. Unified segmentation. Neuroimage. 2005;26:839-851.

27. Patlak CS, Blasberg RG. Graphical evaluation of blood-to-brain transfer constants from multiple-time uptake data: generalizations. J Cereb Blood Flow Metab. 1985;5:584-590.

28. Graham MM, Muzi M, Spence AM, et al. The FDG lumped constant in normal human brain. J Nucl Med. 2002;43:1157-1166.

29. Wienhard K. Measurement of glucose consumption using $\left[{ }^{18} \mathrm{~F}\right]$ fluorodeoxyglucose. Methods. 2002;27:218-225.

30. Poldrack RA, Mumford JA, Nichols TE. Handbook of Functional MRI Data Analysis. New York, NY: Cambridge University Press; 2011.
31. Chen K, Bandy D, Reiman E, et al. Noninvasive quantification of the cerebral metabolic rate for glucose using positron emission tomography, ${ }^{18} \mathrm{~F}$-fluoro-2deoxyglucose, the Patlak method, and an image-derived input function. J Cereb Blood Flow Metab. 1998;18:716-723.

32. Wakita K, Imahori Y, Ido T, et al. Simplification for measuring input function of FDG PET: investigation of 1-point blood sampling method. J Nucl Med. 2000;41: 1484-1490.

33. Sladky R, Baldinger P, Kranz GS, et al. High-resolution functional MRI of the human amygdala at 7T. Eur J Radiol. 2013;82:728-733.

34. Raichle ME, MacLeod AM, Snyder AZ, Powers WJ, Gusnard DA, Shulman GL. A default mode of brain function. Proc Natl Acad Sci USA. 2001;98:676-682.

35. Newberg AB, Wang J, Rao H, et al. Concurrent CBF and CMRGlc changes during human brain activation by combined fMRI-PET scanning. Neuroimage. 2005;28:500-506.

36. Salmi J, Rinne T, Degerman A, Salonen O, Alho K. Orienting and maintenance of spatial attention in audition and vision: multimodal and modality-specific brain activations. Brain Struct Funct. 2007;212:181-194.

37. Striemer CL, Chouinard PA, Goodale MA, de Ribaupierre S. Overlapping neural circuits for visual attention and eye movements in the human cerebellum. Neuropsychologia. 2015;69:9-21.

38. Stoodley CJ, Valera EM, Schmahmann JD. Functional topography of the cerebellum for motor and cognitive tasks: an fMRI study. Neuroimage. 2012;59: 1560-1570.

39. Shulman GL, Corbetta M, Fiez JA, et al. Searching for activations that generalize over tasks. Hum Brain Mapp. 1997;5:317-322.

40. Biswal BB, Mennes M, Zuo X-N, et al. Toward discovery science of human brain function. Proc Natl Acad Sci USA. 2010;107:4734-4739.

41. Logan J. Graphical analysis of PET data applied to reversible and irreversible tracers. Nucl Med Biol. 2000;27:661-670.

42. Witt ST, Laird AR, Meyerand ME. Functional neuroimaging correlates of fingertapping task variations: an ALE meta-analysis. Neuroimage. 2008;42:343-356. 